\title{
Temperature sensor utilizing a ternary photonic crystal with a polymer layer sandwiched between $\mathrm{Si}$ and $\mathrm{SiO}_{2}$ layers
}

\author{
Dena M. El-Amassi ${ }^{1}$. Sofyan A. Taya ${ }^{1}$. Dhasarathan Vigneswaran ${ }^{2}$
}

Received: 22 May 2018 / Accepted: 2 November 2018 / Published online: 14 November 2018

(c) The Author(s) 2018

\begin{abstract}
Recently, one-dimensional periodic ternary photonic crystals have shown outstanding properties when compared to onedimensional periodic binary photonic crystals. In this work, a ternary photonic crystal is proposed as a temperature sensor. The structure of the ternary photonic structure is considered $\mathrm{Si} /$ polymer $/ \mathrm{SiO}_{2}$. The transmission spectra of the proposed ternary photonic structure are studied for different temperatures. It is observed that as the temperature increases, the transmission peak shifts toward higher wavelengths due to thermo-optic and thermal expansion coefficients of the polymer. It is found that the temperature-sensitive transmission peak shift is considerably enhanced due to the insertion of the polymer layer between $\mathrm{Si}$ and $\mathrm{SiO}_{2}$ to constitute a ternary photonic crystal.
\end{abstract}

Keywords Ternary photonic crystal $\cdot$ Temperature sensor $\cdot$ Polymers

\section{Introduction}

The concept of photonic crystals and propagation of electromagnetic waves (EMW) through these structures were introduced since 1987 [1, 2]. One-dimensional photonic crystal has received an increasing interest by many researchers due to the possible use in a considerable set of applications. The most fascinating feature in photonic crystals is the appearance of photonic band gaps in which the transmission of EMW of certain wavelength is not allowed. These structures are constructed by using two or more media repeated alternatively. The width of the band gap varies with the variation of refractive indices of materials, temperature, and the incident angle [3, 4]. If these parameters are maintained fixed, then this photonic structure will have fixed predetermined bands of wavelength or frequencies. Any change in refractive index, temperature, or angle of incidence will change the photonic band gap. This is the rule of operation of a photonic crystal when acting as an optical sensor for detection

Sofyan A. Taya

staya@iugaza.edu.ps

1 Physics Department, Islamic University of Gaza, Gaza, Palestine

2 Department of Electronics and Communication Engineering, Sri Krishna College of Technology, Coimbatore 641 042, India variations in the index of refraction [5-11] and temperature $[12,13]$.

Photonic crystals can be grouped into a set of types according to the number of layers into one period. When there are two layers in a period, it is called binary, and it is known as ternary when there are three layers in a period and so on. In a recent years, photonic crystals have received an increasing attention due to exclusive performance in a set of applications such as omnidirectional reflectors [14], filters [15], and highly sensitive optical sensors [16].

In a recent work, the band gap in a photonic crystal consisting of dielectric/metal/dielectric was shown to be enlarged due to the metal layer [17]. The variation of the band gap width in a periodic photonic crystal with a negative-index material was investigated [18]. An omnidirectional photonic band gap in a photonic crystal having the structure dielectric/plasma layer/dielectric was investigated using the transfer matrix method [19]. The design of 2D photonic crystal-coupled resonating optical waveguidebased integrated optic sensor platform was proposed and investigated [20]. A binary photonic crystal consisting of metal-dielectric layers was analyzed [21]. It was shown that the metal-dielectric photonic crystal can be treated as an effective metallic medium with a well-defined effective plasma frequency [21]. Enhancement of omnidirectional total reflection in a ternary photonic crystal was theoretically proposed [22-24]. 
The rule of operation of a photonic crystal when acting as a temperature sensor is as follows. When the surrounding temperature alters, the index of refraction of substances constituting the crystal will be altered as a result of thermo-optical effect. Moreover, the thickness of the layers in the photonic crystal will be also altered due to the thermal expansion effect. In this case, the transmission profile will be modified. By inspection the transmission spectrum changes or shifts, and a small variation in temperature can be detected. In this way, photonic crystals can be employed as efficient temperature sensors.

In this paper, a ternary photonic crystal with the structure $\mathrm{Si} /$ polymer $/ \mathrm{SiO}_{2}$ is studied as a temperature sensor. The characteristic matrix of a signal cell consisting of three layers is expressed in terms of the refractive indices, thicknesses, wavelength, and angles of refraction inside the media. The variation in refractive index and thickness of the layers due to the variation in temperature is considered. The transfer matrix of the whole system consisting of $\mathrm{N}$-cells is derived, and the transmission coefficient is also deduced. The transmission profile is investigated at different temperatures.

\section{Theory of scattering from a photonic crystal}

One-dimensional ternary photonic crystal is assumed. It consists of three layers of indices $n_{1}, n_{2}$, and $n_{3}$. The layers are assumed to have the thicknesses $d_{1}, d_{2}$, and $d_{3}$. If we assume one period consisting of three layers, then the thickness of the period will be $d=d_{1}+d_{2}+d_{3}$.

Thickness of a substance $(d)$ can be altered as a result of thermal expansion effect according to

$\Delta d=\alpha d(\Delta T)$

where $\alpha$ is the thermal expansion coefficient of the substance and $\Delta T$ is the change in temperature.

Refractive index a substance $(n)$ can be also altered as a result of thermo-optical effect according to

$\Delta n=\gamma n(\Delta T)$

where $\gamma$ is the coefficient of thermo-optic of a substance.

Consider an EMW is incident from air on the proposed photonic crystal. The incident angle is assumed $\theta_{0}$. The transmission of the wave can be calculated by making use of the transfer matrix method. The transfer matrix of a single layer is given by:

$h_{i}=\left[\begin{array}{cc}\cos \beta_{i} & \frac{-i \sin \beta_{i}}{p_{i}} \\ -i p_{i} \sin \beta_{i} & \cos \beta_{i}\end{array}\right]$.

The characteristic matrix $L[d]$ of a signal period is expressed as:
$L[d]=\prod_{i=1}^{s}\left[\begin{array}{cc}\cos \beta_{i} & \frac{-i \sin \beta_{i}}{p_{i}} \\ -i p_{i} \sin \beta_{i} & \cos \beta_{i}\end{array}\right]=\left[\begin{array}{ll}L_{11} & L_{12} \\ L_{21} & L_{22}\end{array}\right]$,

where $s=3$ and $i=1,2$, and 3 denote the layers of refractive indices $n_{1}, n_{2}$, and $n_{3}$, respectively, and $\beta_{i}$ and $p_{i}$ are given as

$\beta_{1}=\frac{2 \pi\left(n_{1}+\Delta n_{1}\right)\left(d_{1}+\Delta d_{1}\right) \cos \theta_{1}}{\lambda_{0}}$,

$\beta_{2}=\frac{2 \pi\left(n_{2}+\Delta n_{2}\right)\left(d_{2}+\Delta d_{2}\right) \cos \theta_{2}}{\lambda_{0}}$,

$\beta_{3}=\frac{2 \pi\left(n_{3}+\Delta n_{3}\right)\left(d_{3}+\Delta d_{3}\right) \cos \theta_{3}}{\lambda_{0}}$,

$p_{1}=\left(n_{1}+\Delta n_{1}\right) \cos \theta_{1}$,

$p_{2}=\left(n_{2}+\Delta n_{2}\right) \cos \theta_{2}$,

$p_{3}=\left(n_{3}+\Delta n_{3}\right) \cos \theta_{3}$,

with $\lambda_{0}$ is the wavelength in vacuum. Angles $\theta_{1}, \theta_{2}$, and $\theta_{3}$ are the angles of refraction in the three layers of the cell. In terms of $\theta_{0}$, they are given by:

$\cos \theta_{1}=\left[1-\frac{n_{0}^{2} \sin ^{2} \theta_{0}}{\left(n_{1}+\Delta n_{1}\right)^{2}}\right]^{\frac{1}{2}}$,

$\cos \theta_{2}=\left[1-\frac{n_{0}^{2} \sin ^{2} \theta_{0}}{\left(n_{2}+\Delta n_{2}\right)^{2}}\right]^{\frac{1}{2}}$,

$\cos \theta_{3}=\left[1-\frac{n_{0}^{2} \sin ^{2} \theta_{0}}{\left(n_{3}+\Delta n_{3}\right)^{2}}\right]^{\frac{1}{2}}$.

The determinant of the matrix $L[d]$ is equal to unity.

For a system consisting of $N$ periods, the characteristic matrix is given by:

$$
\begin{aligned}
{[L(d)]^{N} } & =\left[\begin{array}{ll}
L_{11} T_{N-1}(a)-T_{N-2}(a) & L_{12} T_{N-1}(a) \\
L_{21} T_{N-1}(a) & L_{22} T_{N-1}(a)-T_{N-2}(a)
\end{array}\right] \\
& =\left[\begin{array}{ll}
s_{11} & s_{12} \\
s_{21} & s_{22}
\end{array}\right]
\end{aligned}
$$

where

$s_{11}=\left(\sigma_{1}-\frac{p_{2}}{p_{1}} \sigma_{2}-\frac{p_{3}}{p_{2}} \sigma_{3}-\frac{p_{3}}{p_{1}} \sigma_{4}\right)$, 
$s_{12}=-i\left(\frac{\rho_{1}}{p_{1}}+\frac{\rho_{2}}{p_{2}}+\frac{\rho_{3}}{p_{3}}-\frac{p_{2} \rho_{4}}{p_{1} p_{2}}\right)$,

$s_{21}=-i\left(p_{1} \rho_{1}+p_{2} \rho_{2}+p_{3} \rho_{3}-\frac{p_{1} p_{3}}{p_{2}} \rho_{4}\right)$,

$s_{22}=\left(\sigma_{1}-\frac{p_{1}}{p_{2}} \sigma_{2}-\frac{p_{2}}{p_{3}} \sigma_{3}-\frac{p_{1}}{p_{3}} \sigma_{4}\right)$.

where $\sigma_{1}=\cos \beta_{1} \cos \beta_{2} \cos \beta_{3}, \sigma_{2}=\sin \beta_{1} \sin \beta_{2} \cos \beta_{3}$, $\sigma_{3}=\cos \beta_{1} \sin \beta_{2} \sin \beta_{3}, \sigma_{4}=\sin \beta_{1} \cos \beta_{2} \sin \beta_{3}$, $\rho_{1}=\sin \beta_{1} \cos \beta_{2} \cos \beta_{3}, \rho_{2}=\cos \beta_{1} \sin \beta_{2} \cos \beta_{3}$, $\rho_{3}=\cos \beta_{1} \cos \beta_{2} \sin \beta_{3}$ and $\rho_{4}=\sin \beta_{1} \sin \beta_{2} \sin \beta_{3}$.

$T_{N}$ are Chebyshev polynomials of the second kind given by:

$T_{N}(a)=\frac{\sin \left[(N+1) \cos ^{-1} a\right]}{\left[1-a^{2}\right]^{\frac{1}{2}}}$,

and $a$ is given by

$a=\frac{1}{2}\left[s_{11}+s_{22}\right]$.

In terms of the elements $s_{i j}$, the transmission coefficient of a system consisting of $N$ periods is given by:

$t=\frac{2 p_{0}}{\left(s_{11}+s_{12} p_{0}\right) p_{0}+\left(s_{21}+s_{22} p_{0}\right)}$, where

$p_{0}=n_{0} \wedge Z \cos \theta_{0}=\cos \theta_{0} \quad\left(\right.$ as $\left.n_{0}=1\right)$

The transmissivity of the system can be written as:

$T=|t|^{2}$.

\section{Results and discussion}

Ternary photonic crystal is considered of the structure $\mathrm{Si} /$ polymer/SiO ${ }_{2}$. In Fig. 1, we consider the polymer to be polycarbonate. The parameters of the structure are taken as [13] $n_{1}=3.3(\mathrm{Si}), n_{3}=1.46\left(\mathrm{SiO}_{2}\right), d_{1}=117 \mathrm{~nm}$, and $d_{3}=265 \mathrm{~nm}$. The number of the period is considered $N=10$. The coefficient of thermo-optic $(\gamma)$ of a material represents the change in the index of refraction per degree rise in temperature, whereas the coefficient of thermal expansion $(\alpha)$ of a material represents the change in the dimensions of a material per degree rise in temperature. In the following calculations, we take $\gamma=1.86 \times 10^{-4}{ }^{\circ} \mathrm{C}^{-1}(\mathrm{Si}), \gamma=6.8 \times 10^{-6}{ }^{\circ} \mathrm{C}^{-1}$ $\left(\mathrm{SiO}_{2}\right), \alpha=0.5 \times 10^{-6}{ }^{\circ} \mathrm{C}^{-1}(\mathrm{Si})$ and $\alpha=2.6 \times 10^{-6}{ }^{\circ} \mathrm{C}^{-1}$ $\left(\mathrm{SiO}_{2}\right)$. The parameters of polycarbonate are taken as $n_{2}=1.5848$ and $d_{2}=10 \mathrm{~nm}$. In a recent study conducted by Zhang et al. [23] on polymers to determine their thermal coefficients for optical waveguide applications, they found that $\gamma=0.9 \times 10^{-4}{ }^{\circ} \mathrm{C}^{-1}$ and $\alpha=1.7 \times 10^{-6}{ }^{\circ} \mathrm{C}^{-1}$ for polycarbonate. The angle of incidence is considered to be $\theta_{0}=0$ (normal incidence). In Fig. 1, the transmission spectra of one-dimensional ternary photonic crystal structure are
Fig. 1 Transmission spectra of one-dimensional ternary photonic crystal structure with $n_{1}=3.3(\mathrm{Si}), n_{2}=1.5848$ (polycarbonate), $n_{3}=1.46\left(\mathrm{SiO}_{2}\right)$, $d_{1}=117 \mathrm{~nm}, d_{2}=10 \mathrm{~nm}$ and $d_{3}=265 \mathrm{~nm}, N=10$ at temperatures of $25^{\circ} \mathrm{C}, 50^{\circ} \mathrm{C}, 75^{\circ} \mathrm{C}$, and $100^{\circ} \mathrm{C}$
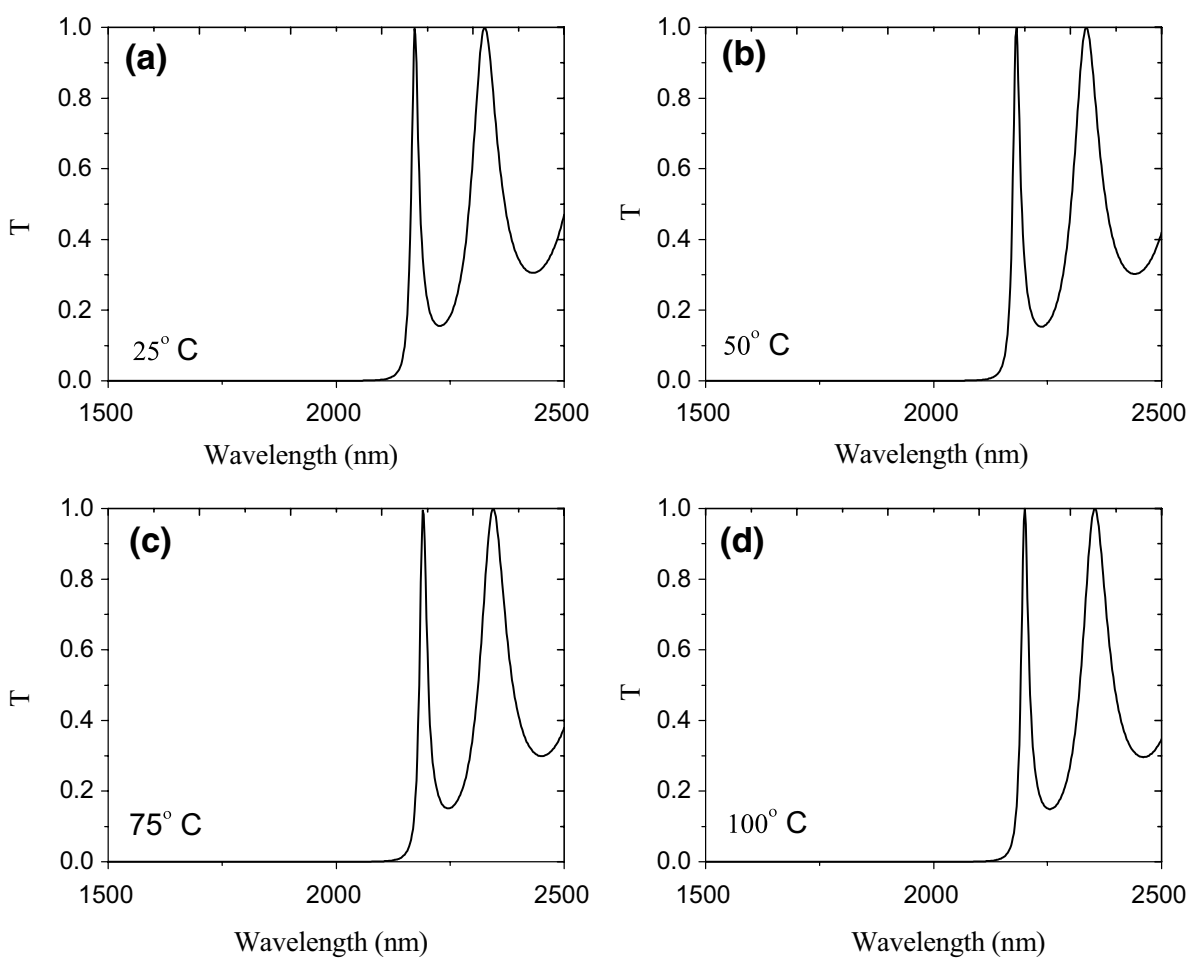
shown for temperatures of $25^{\circ} \mathrm{C}, 50{ }^{\circ} \mathrm{C}, 75^{\circ} \mathrm{C}$, and $100{ }^{\circ} \mathrm{C}$. Inspecting the first transmission peak at different temperatures, we find that the wavelengths corresponding to the first peak are $\lambda_{1}=2172 \mathrm{~nm}\left(25^{\circ} \mathrm{C}\right), \lambda_{1}=2181.2 \mathrm{~nm}\left(50^{\circ} \mathrm{C}\right)$, $\lambda_{1}=2190.4 \mathrm{~nm}\left(75^{\circ} \mathrm{C}\right)$, and $\lambda_{1}=2199.6 \mathrm{~nm}\left(100^{\circ} \mathrm{C}\right)$. This means that for a temperature rise of $75^{\circ} \mathrm{C}$, we have a transmission peak shift of $27.6 \mathrm{~nm}$. Then we have a transmission peak shift of $0.368 \mathrm{~nm}$ degree rise in temperature. It is worth mentioning that the shift occurs toward higher wavelengths. Moreover, the shift versus temperature of the first peak is shown in Fig. 2 for the photonic crystal containing polycarbonate. It is clear that $\Delta \lambda / \Delta T=0.368$.

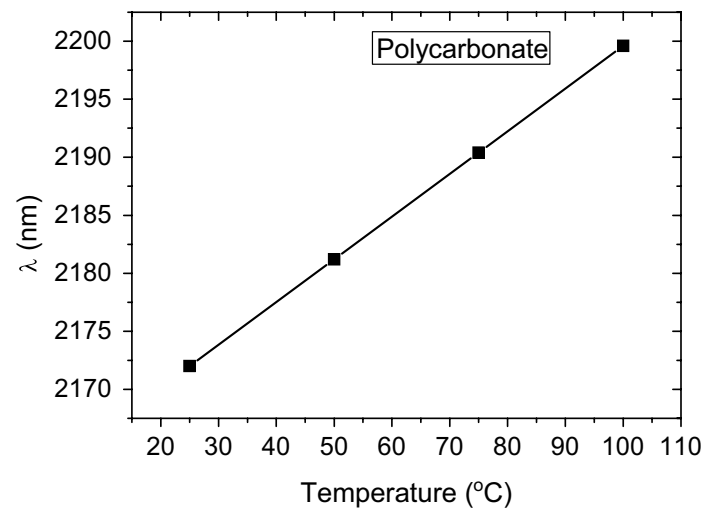

Fig. 2 Shift versus temperature of the first peak for polycarbonate
In Fig. 3, the same parameters as in Fig. 1 are adopted. The only difference between them is the polymer. We consider in Fig. 3 the polymer to be polystyrene of index of refraction $\mathrm{n}_{2}=1.5916$. From Ref. [25], they found $\gamma=1.2 \times 10^{-4}{ }^{\circ} \mathrm{C}^{-1}$ and $\alpha=2.2 \times 10^{-6}{ }^{\circ} \mathrm{C}^{-1}$ for polystyrene. The transmission spectra of the proposed ternary photonic crystal with polystyrene layer are shown in Fig. 2 for the same values of temperature. As can be seen from the figure, the first transmission peak are found to occur at $\lambda_{1}=2172 \mathrm{~nm}$ $\left(25^{\circ} \mathrm{C}\right), \lambda_{1}=2181.5 \mathrm{~nm}\left(50^{\circ} \mathrm{C}\right), \lambda_{1}=2191 \mathrm{~nm}\left(75^{\circ} \mathrm{C}\right)$ and $\lambda_{1}=2200.5 \mathrm{~nm}\left(100{ }^{\circ} \mathrm{C}\right)$. For a temperature rise of $75^{\circ} \mathrm{C}$, there is a transmission peak shift of $28.5 \mathrm{~nm}$. A transmission peak shift of $0.380 \mathrm{~nm}$ per degree rise in temperature. There is a preference of using polystyrene over polycarbonate because of the higher transmission peak shift.

We also show the shift versus temperature of the first peak in Fig. 4 for the photonic crystal containing polystyrene. It is clear that $\Delta \lambda / \Delta T=0.380$.

It is worth mentioning that glass transition points of polycarbonate and polystyrene are $147{ }^{\circ} \mathrm{C}$ and $100{ }^{\circ} \mathrm{C}$, respectively. Melting points of the two polymers are $225^{\circ} \mathrm{C}$ for polycarbonate and $240{ }^{\circ} \mathrm{C}$ for polystyrene. So the proposed sensor cannot be used for temperatures higher than $100{ }^{\circ} \mathrm{C}$ especially that consisting of $\mathrm{Si} /$ polystyrene/ $/ \mathrm{SiO}_{2}$. If it is used for sensing high temperatures, shrinking of the layers may occur.

In a recent study [12], A. Banerjee compared binary and ternary photonic crystals as temperature sensors. He found that a temperature peak shift of $0.350 \mathrm{~nm}$ can be obtained
Fig. 3 Transmission spectra of one-dimensional ternary photonic crystal structure with $n_{1}=3.3(\mathrm{Si}), n_{2}=1.5916$ (Polystyrene), $n_{3}=1.46\left(\mathrm{SiO}_{2}\right)$, $d_{1}=117 \mathrm{~nm}, d_{2}=10 \mathrm{~nm}$ and $d_{3}=265 \mathrm{~nm}, N=10$ at temperatures of $25^{\circ} \mathrm{C}, 50^{\circ} \mathrm{C}, 75^{\circ} \mathrm{C}$ and $100{ }^{\circ} \mathrm{C}$
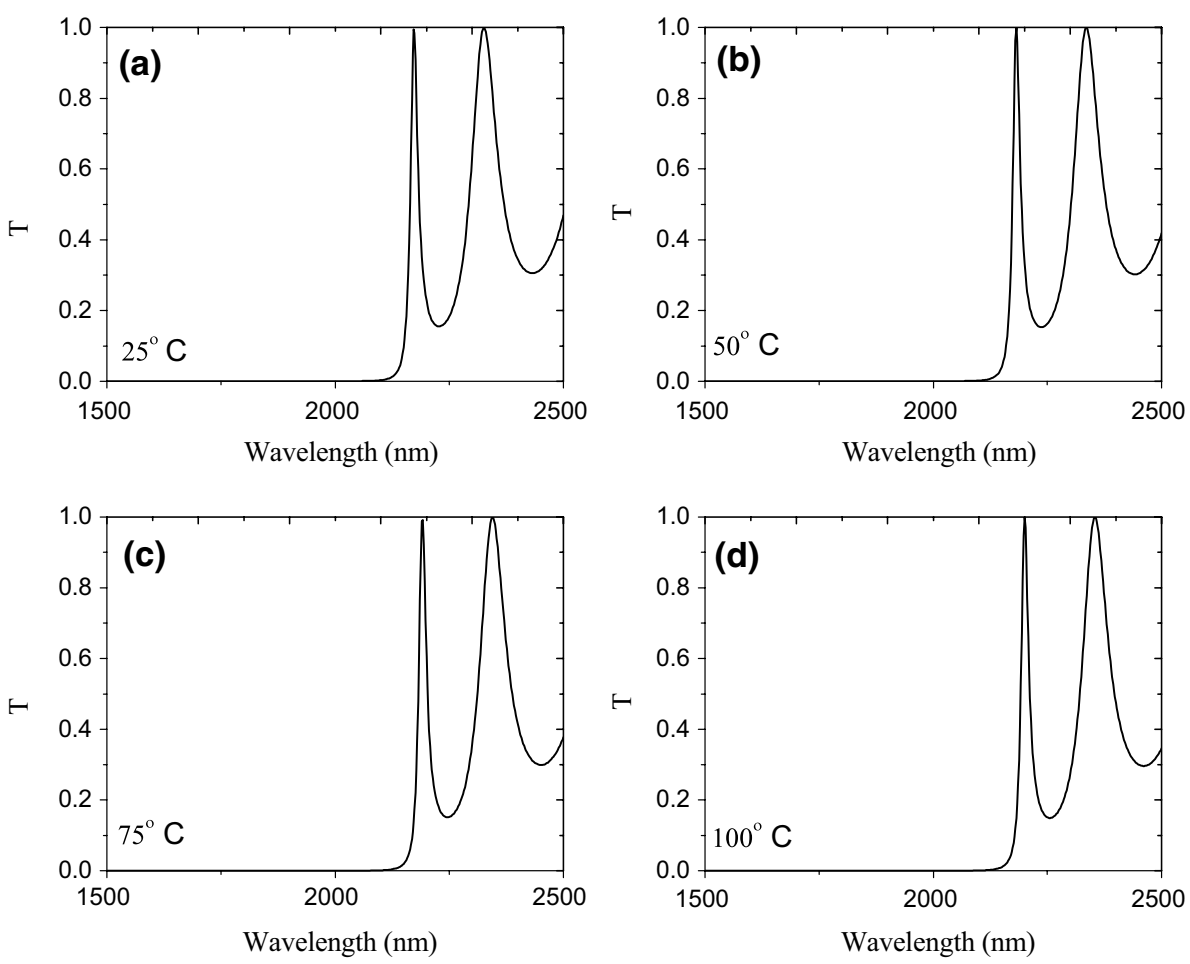


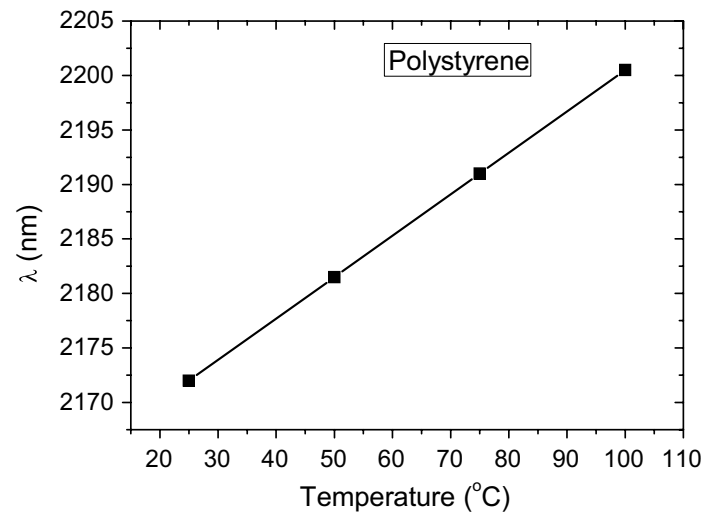

Fig. 4 Shift versus temperature of the first peak for polystyrene

with the binary structure, whereas a peak shift of 0.355 can be attained with the ternary one. In his structure, he used $\mathrm{Bi}_{4} \mathrm{Ge}_{3} \mathrm{O}_{12}$ to form a ternary photonic crystal.

In this work, a much higher peak shift is obtained with the use of polymers (polycarbonate and polystyrene) instead of $\mathrm{Bi}_{4} \mathrm{Ge}_{3} \mathrm{O}_{12}$.

\section{Conclusion}

Recently, binary and ternary photonic crystals were proposed in temperature sensing. The author of Ref. [12] found that with a binary photonic crystal, a temperature peak shift of $0.350 \mathrm{~nm}$ may be obtained, whereas with a ternary photonic crystal, a peak shift of 0.355 may be obtained. His ternary photonic crystal has a working layer of $\mathrm{Bi}_{4} \mathrm{Ge}_{3} \mathrm{O}_{12}$. No doubt that this was a fascinating result. In this work, a much higher peak shift was obtained using a polymer as a working layer. The proposed ternary photonic crystal was assumed to have the structure $\mathrm{Si} /$ poly$\mathrm{mer} / \mathrm{SiO}_{2}$. Transmission profile of this photonic structure was investigated at different temperatures. It was found that with the increase in temperature, the transmission peak shifts toward higher wavelengths. This can be attributed to thermo-optic and thermal expansion coefficients. When the polymer "polycarbonate" was used, a transmission peak shift of $0.368 \mathrm{~nm}$ per degree rise in temperature was obtained, whereas when the polymer (Polystyrene) was employed, a transmission peak shift of $0.380 \mathrm{~nm}$ per degree rise in temperature.

It is concluded that the temperature-sensitive transmission peak shift is considerably enhanced due to the insertion of the polymer layer between $\mathrm{Si}$ and $\mathrm{SiO}_{2}$ to constitute a ternary photonic crystal.

Finally, it is worth mentioning that a ternary photonic crystal of $\mathrm{Si} /$ polymer $/ \mathrm{SiO}_{2}$ would involve a complicated sputtering/spin coating/sputtering procedure for $\mathrm{N}$ periods. In future work, we may try to find alternative transparent inorganic materials of appropriate properties.

Open Access This article is distributed under the terms of the Creative Commons Attribution 4.0 International License (http://creativecommons.org/licenses/by/4.0/), which permits unrestricted use, distribution, and reproduction in any medium, provided you give appropriate credit to the original author(s) and the source, provide a link to the Creative Commons license, and indicate if changes were made.

\section{References}

1. Yablonovitch, E.: Inhibited spontaneous emission in solid state physics and electronics. Phys. Rev. Lett. 58, 2059-2062 (1987)

2. John, S.: Strong localization of photons in certain disordered dielectric superlattices. Phys. Rev. Lett. 58, 2486-2489 (1987)

3. Kumar, V., Suthar, B., Kumar, A., Singh, KhS, Bhargava, A.: The effect of temperature and angle of incidence on photonic band gap in a dispersive Si-based one dimensional photonic crystal. Phys. B-Condens. Matter 416, 106-109 (2013)

4. Suthar, B., Kumar, V., Kumar, A., Singh, KhS, Bhargava, A.: Thermal expansion of photonic bandgap for 1-D photonic crystals. Prog. Electromagn. Res. Lett. 32, 81-90 (2012)

5. Shaheen, S.A., Taya, S.A.: Propagation of p-polarized light in photonic crystal for sensor application. Chin. J. Phys. 55, 571-582 (2017)

6. Taya, S.A., Shaheen, S.A., Alkanoo, A.A.: Photonic crystal as a refractometric sensor operated in reflection mode. Superlattices Microstruct. 101, 299-305 (2017)

7. Alkanoo, A.A., Taya, S.A.: Theoretical investigation of fivelayer waveguide structure including two left-handed material layers for refractometric applications. J. Magn. Magn. Mater 449, 395-400 (2018)

8. Taya, S.A., Mahdi, S.S., Alkanoo, A.A., Qadoura, I.M.: Slab waveguide with conducting interfaces as an efficient optical sensor: TE case. J. Mod. Opt. 64, 836-843 (2017)

9. Taya, S.A.: Theoretical investigation of slab waveguide sensor using anisotropic metamaterials. Opt. Appl. 45, 405-417 (2015)

10. Taya, S.A.: P-polarized surface waves in a slab waveguide with left-handed material for sensing applications. J. Magn. Magn. Mater 377, 281-285 (2015)

11. Taya, S.A.: Slab waveguide with air core layer and anisotropic left-handed material claddings as a sensor. Opto-Electron Rev. 22, 252-257 (2014)

12. Banerjee, A.: Enhanced temperature sensing by using onedimensional ternary photonic band gap structures. Prog. Electromagn. Res. Lett. 11, 129-137 (2009)

13. Kai, T., Wei, C.W., Hui, Y.G., Quan, L.Z.: Study on temperature property of band structures in one-dimensional photonic crystals. Optoelectron. Lett. 3, 0444-0447 (2007)

14. Awasthi, S.K., Malaviya, U., Ojha, S.P.: Enhancement of omnidirectional total-reflection wavelength range by using onedimensional ternary photonic bandgap material. J. Opt. Soc. Am. B 23, 2566-2571 (2006)

15. Awasthi, S.K., Ojha, S.P.: Design of a tunable optical filter by using one-dimensional ternary photonic band gap material. Prog. Electromagn. Res. M 4, 117-132 (2008)

16. Banerjee, A.: Enhanced refractometric optical sensing by using one-dimensional ternary photonic crystals. Prog. Electromagn. Res. PIER 89, 11-22 (2009) 
17. Wu, C.J., Chung, Y.H., Syu, B.J.: Band gap extension in a onedimensional ternary metal-dielectric photonic crystal. Prog. Electromagn. Res. PIER 102, 81-93 (2010)

18. Zare, L.Z., Gharaati, A.: Investigation of band gap width in ternary 1D photonic crystal with left-handed. Acta Phys. Pol. A 125, 36-38 (2014)

19. Xue, F., Liu, S.B., Zhang, H.F., Kong, X.K., Wen, Y.D., Wang, L.L., Qian, S.: The theoretical analysis of omnidirectional photonic band gaps in the one-dimensional ternary plasma photonic crystals based on Pell quasi-periodic structure. Opt. Quant. Electron 49, 19 (2017)

20. Goyal, A.K., Dutta, H.S., Pal, S.: Performance optimization of photonic crystal resonator based sensor. Opt. Quant. Electron. 48, 431 (2016)

21. Xu, X., Xi, Y., Han, D., Liu, X., Zi, J., Zhu, Z.: Effective plasma frequency in one-dimensional metallic-dielectric photonic crystals. Appl. Phys. Lett. 86, 091112 (2005)

22. Awasthi, S.K., Malaviya, U., Ojha, S.P.: Enhancement of omnidirectional total-reflection wavelength range by using one-dimensional ternary photonic bandgap material. JOSA B $\mathbf{2 3}$, 2566-2571 (2006)

23. Dai, X., Xiang, Y., Wen, S.: Broad omnidirectional reflector in the one-dimensional ternary photonic crystals containing superconductor. Prog. Electromagn. Res. 120, 17-34 (2011)

24. Bellingeri, M., Chiasera, A., Kriegel, I., Scotognella, F.: Optical properties of periodic, quasi-periodic, and disordered onedimensional photonic structures. Opt. Mater 72, 403-421 (2017)

25. Zhang, Z., Zhao, P., Lin, P., Sun, F.: Thermo-optic coefficients of polymers for optical waveguide applications. Polymer 47, 48934896 (2006)

Publisher's Note Springer Nature remains neutral with regard to jurisdictional claims in published maps and institutional affiliations. 